\title{
Clinical Medication Review and Falls in Older People-What Is the Evidence Base?
}

\author{
N. Tanna1,2, T. Tatla1, T. Winn'1, S. Chita1, K. Ramdoo1, C. Batten1, J. Pitkin'1,2 \\ ${ }^{1}$ London North West Healthcare NHS Trust, London, UK \\ ${ }^{2}$ School of Science \& Medicine, Imperial College, London, UK \\ Email: nuttantanna@nhs.net
}

Received 16 December 2015; accepted 19 February 2016; published 22 February 2016

Copyright (C) 2016 by authors and Scientific Research Publishing Inc.

This work is licensed under the Creative Commons Attribution International License (CC BY). http://creativecommons.org/licenses/by/4.0/

(c) (i) Open Access

\section{Abstract}

Background: This paper reports findings from a literature review undertaken to assess the current evidence base for clinical medication review and falls in older people. This forms part of a larger, organisational supported project design work-stream, where the objectives are to define the operational details for clinical medication review as part of multi-factorial assessment for elderly fallers in the community. Patients will be identified and targeted through an integrated care pathway mapping and elderly patient care screening service. Objective: A review of national and best practice guidance to help our understanding of how clinical medication review could be optimised. Methods: A PubMed database search was undertaken with search terms including "elderly" and "falls" and "medicines" followed by study of relevant publications in English and including cited referenced publications within selected papers. Results: Our findings were that both medication over-use and under-use in the elderly occur frequently and can be harmful. Many drugs commonly used by older persons have not been systematically studied as risk factors for falls. The screening tool of older people's prescriptions (STOPP) and screening tool to alert to right treatment (START), validated for assessment of potentially inappropriate prescribing in the elderly, offer the possibility of provision of a structured clinical medication review to patients, with a need for more research on the impact of the STOPP START interventions on both the rates of falls and risk of falls in the elderly.

\section{Keywords}

Clinical Medication Review, Falls, Elderly, Medicines, Multi-Factorial Interventions

\section{Introduction}

Falls are a common, costly and preventable consequence of ageing [1] [2]. Older people are susceptible to fal-

How to cite this paper: Tanna, N., Tatla, T., Winn, T., Chita, S., Ramdoo, K., Batten, C. and Pitkin, J. (2016) Clinical Medication Review and Falls in Older People-What Is the Evidence Base? Pharmacology \& Pharmacy, 7, 89-96. 
ling due to an age-related decline in the sensory and neuromuscular systems that contribute to postural stability. A fall occurs when the physical ability of the individual is unable to match the immediate demands of the environment and/or of the activity being undertaken and is defined as an event which results in a person coming to rest inadvertently on the ground or floor or other lower levels [2]. Around 30\% of people aged 65 and over living in the community and more than $50 \%$ of those living in residential care facilities or nursing homes fall every year, and about half of those who fall do so repeatedly [2]-[6].

Factors increasing an older person's risk of falling include advanced age, reduced leg strength, balance deficits, history of falling, culprit medication use, and visual and cognitive impairment [5]. Lin and Ferrucci [7] have also noted that people with hearing loss and balance problems experience problems with walking and mobility. Sensory impairment and age related hearing loss (presbyacusis) may add to the number of falls sustained [8] [9]. In addition, symptoms of hearing loss and dementia maybe confused, with a combination likely to increase the number and severity of symptoms experienced by patients [10].

In 2012 the World Health Organisation reported "falls" as the second leading cause of accidental or unintentional injury deaths worldwide [2]. Injuries received from unintentional falls result in death, disability, nursinghome admission and direct medical costs [4]. Although certain interventions can reduce falls (e.g., exercising regularly or having medicines reviewed to reduce side effects and interactions), implementation at the community level remains limited and additional measures are needed to promote widespread adoption [4] [11] [12]. Recent guidance to support the care of older people living with frailty in the community or outpatient settings [13] stated that "falls" and "susceptibility to side effects of medicines" are two presentations that should raise suspicion that an individual has frailty that may mask more serious underlying disease.

\section{Objectives}

The objectives for this paper are to assess what the current evidence base is to support a clinical medication review service to reduce falls risk and falls related injuries in older people in the community setting. The evidence base will be utilised in the next phase of this work-stream to help define the operational details for providing a clinical medication review service to elderly fallers as part of an integrated care pathway mapping and multidisciplinary elderly patient care screening service. The pathway will also include assessment for early cognitive impairment, hearing loss, vision and balance as part of a Falls Care Bundle implementation approach [14] to ensure a high standard of quality health care delivery.

\section{Background: Optimising Clinical Medication Review}

Care facilities and hospitals setting: A recent Cochrane Database review [6] assessed the effectiveness of interventions designed to reduce falls by older people in care facilities and hospitals. 43 randomised controlled trials with 30,373 participants in care facilities were included in this systematic review with the authors noting that despite the large number of trials there was limited evidence to support any one intervention. Rate of falls were calculated as falls per person year with risk of falling classified as the number of people falling (fallers) in each group.

Results from 13 trials testing exercise interventions in care facilities were inconsistent; exercise programmes seem to increase falls in frail residents in nursing homes, but reduce falls in people in intermediate care facilities. For multi-factorial interventions in care facilities, both the rate of falls and risk of falls showed a trend towards possible benefit. Multi-factorial interventions [15] usually include medication review, but current published literature does not define a process for a structured clinical medication review. The British Geriatric Society in their recent guidance [13] recommend that evidence based medication reviews should be conducted for older people with frailty, and cite the use of STOPP START criteria [6] [17] as an example.

Considering trials for specific medicines, Cameron et al. [6] noted that Vitamin D supplementation was effective in reducing the rate of falls, but not the risk of falling. Bjelakovic et al. [18] have reported findings from their updated Cochrane Database review where the objective was to assess the beneficial and harmful effects of Vitamin D supplementation for prevention of mortality in healthy adults and adults in a stable phase of disease. Including 56 trials with 95,286 participants providing usable data on mortality, the authors noted that Vitamin D3 may decrease mortality in elderly people living independently or in institutionalised care, but there was a need for further evidence from placebo controlled randomised trials in this area.

Community setting: Community dwelling older individuals are a large group identified as at high risk of fu- 
ture falls and injuries [1]. Many are subsequently admitted into intermediate care facilities.

Gillespie et al. [19] reviewed the interventions for preventing falls in older people living in the community, with this review including 159 trials with 79,193 participants. The most common interventions tested were exercise as a single intervention (59 trials) and multi-factorial programmes (40 trials). Kannus et al. [3] suggest that a multi-factorial intervention for elderly people is more effective than its single intervention counterpart since "causes and risk factors of falling are usually multiple with striking intra-individual (fall to fall) and interindividual variation". The Cochrane review [19] found that multi-factorial interventions which included individualised risk assessment reduced rate of falls but not risk of falling. Trials looking at medication review did not demonstrate benefit but a prescribing modification programme also involving primary care physicians and their patients significantly reduced risk of falling. The Cochrane review [19] also found that gradual withdrawal of psychotropic medication reduced rate of falls but not risk of falling.

\section{Methods}

A PubMed database literature review was undertaken. Search strategy terms used were "elderly" and "falls" and "medicines", with the search undertaken in and to July 2014, with selection criteria including review of all abstracts (NT), followed by review of selected publications in English and any relevant cited references within selected publications, critiqued by three members of the research group (TT, JP and CB). Including the search term "older" in the search strategy did not elicit any further publications of relevance.

\section{Results with Discussion}

An observational study in older men living in the community [20] found that a self-reported history of falls in the previous 12 months was independently associated with number of medicines taken (odds ratio [OR] $=1.06$, $95 \%$ confidence interval [CI] 1.02, 1.09) and use of one or more potentially inappropriate medicines (PIMs) $(\mathrm{OR}=$ $1.23,95 \%$ CI 1.04, 1.45). Use of one or more PIMs had a correlation with hospital admission (hazard ration $[\mathrm{HR}]=1.16,95 \%$ CI 1.08, 1.24), whilst potential under-utilization was associated with cardiovascular events $(\mathrm{HR}=1.20,95 \% \mathrm{CI} 1.03,1.40)$, with the authors concluding that both medication over-use and under-use occur frequently among older men and may be harmful.

Gnjidic et al. [21] studied the optimal discriminating number of concomitant medications that were associated with geriatric syndromes, functional outcomes, and mortality in their study population of community-dwelling older men. They validated this as the use of five or more medications, accepted as the current definition of polypharmacy, and found this to be helpful in estimating the medication-related adverse effects for frailty, disability, mortality, and falls.

The aims for a 2007 critical systematic review [22] were to include all original articles examining medication use as a risk factor for falls or fall-related fractures in people aged 60 years or older with findings reported after the research group had assessed 28 observational studies and one randomized controlled trial. The number of participants in the trials ranged from 70 to 132,873. The outcome measure was a fall in 22 studies and a fracture in 7 studies. The authors found that the main group of drugs associated with an increased risk of falling were psychotropics, including benzodiazepines, antidepressants, and antipsychotics (Table 1). Antiepileptics and drugs that lower blood pressure were weakly associated with falls. However limitations included a need for improvement in the quality of observational studies, as many did not have a clear definition of a fall or target medicines, or prospective follow-up. The researchers concluded that many drugs commonly used by older persons are not systematically studied as risk factors for falls.

A recent Australian study [23] undertook a retrospective analysis of the management of pain control and reported that over $90 \%$ of residents in aged care facilities (ACF) were prescribed analgesics. Of those, 2057 residents were taking regular opioids (28.1\%). Only $50 \%$ of those taking regular opioids received regular paracetamol at doses of $3-4 \mathrm{~g} /$ day. The concurrent use of sedatives was high, with $48.4 \%$ of those taking regular opioids also taking an anxiolytic/hypnotic. With the risk of falls and fractures increased by concurrent use of opioids and sedatives, the widespread use of these drugs in a population already at high risk was concerning. The researchers commented on the need to optimise the prescribing and administration of regular paracetamol as a first line and continuing therapy for pain management in ACF residents, to potentially improve pain management and reduce opioid requirements.

Nishtala et al. [24] reported findings from a large multi-database study that assessed the impact of polypharmacy 
- Psychotropics and cardiovascular drugs associated with increased risk of falling.

o Risk of falls and fractures increases with concurrent use of opioids and sedatives.

o Poly-pharmacy and cumulative exposure to anticholinergic and sedative medicines associated with fall related hospitalizations.

- Need to optimise prescribing and administration of regular Paracetamol as first line and continuing therapy for pain management.

- Recent changes in psychotropic and cardiovascular medications associated with substantial increase in risk of hospital admission for falls and fractures.

- Use of psychotropic medication in females associated with increased risk of falls and fractures, and body mass index (BMI) is a confounding variable.

and exposure to Drug Burden Index (DBI) medicines, with exposure defined by the researchers as quantification of each individual's cumulative exposure to anticholinergic and sedative medicines. Both criteria were independently associated with fall-related hospitalisations, frequency of GP visits, and risk of mortality. DBI drugs were associated with fall-related hospitalisations with an incidence rate ratio (IRR) of 1.56 (95\% CI $=1.47$ 1.65) and greater number of GP visits (IRR 1.13, 95\% CI = $1.12-1.13$ ).

Another study [25] evaluated the prevalence of adverse drug events in an acute geriatric setting over a 6 month period and reported a rate of $12.7 \%(\mathrm{n}=313$; mean age 84.8). Cardiovascular (39\%), psychotropic (36.6\%) and opiate (7.3\%) medicines were the most frequently involved. The adverse events that occurred most frequently were bleeding (28.6\%), falls (14.3\%), and sleepiness $(9.5 \%)$, with the authors noting that these could have been "prevented" in 31\% of cases. Preventability by the research group was determined by assessment of inadequacy with standards of care, medication-related factors (excluding contra-indication) and use of standard lists of harmful medication in the elderly.

A French retrospective case control study [26] reported that $50 \%$ of falls occurred in patients in their first week after hospital admission, with these classified as severe in $16 \%$ cases. The characteristics of the two groups under study (patients who fell and those who did not) were similar: there were no significant differences in variables such as age, sex, number of medicines or prevalence of hypertension or Parkinson's disease. Probability of falls increased when the patients used zolpidem (adjusted odds ratio [AOR] 2.59; 95\% CI 1.16, 5.81; $\mathrm{p}=$ 0.02), meprobamate (AOR 3.01; 95\% CI 1.36, 6.64; $\mathrm{p}=0.01$ ) or calcium channel antagonists (AOR 2.45; 95\% CI 1.16, 4.74; $\mathrm{p}=0.02$ ).

Payne et al. reported data from a larger Scottish retrospective case-cohort study [27] which included over 39,000 patients aged over 65 years. They found that the period with recent changes in psychotropic and cardiovascular medications was associated with a substantial increase in risk of hospital admission for falls and fractures; with an odds ratio of 1.54 (95\% CI 1.17 - 2.03) and 1.68 (95\% CI 1.28 - 2.22) respectively (Table 1). These findings are in line with work reported by Beer et al. [20] with correlation both for falls risk and hospital admission with use of potentially inappropriate medicines. Notably Payne et al. (27) found evidence $(\mathrm{p}=0.003)$ for variation in the association between change in different psychotropic medications and admission, with the strongest associations for changes with selective serotonin reuptake inhibitor (SSRI) antidepressants (OR 1.99 [95\% CI 1.29 - 3.08]), non-SSRI/tricyclic antidepressants (OR 4.39 [95\% CI 2.21 - 8.71]) and combination psychotropic medication (OR 3.05 [95\% CI 1.66 - 5.63]).

The Australian Longitudinal Study of Ageing [28] specifically looked at the risk of falls with use of psychotropic medicines. Interestingly, use was associated with increased risk of falls in females (IRR $=1.47,95 \% \mathrm{CI}=$ 1.31 - 1.64) but not in males ( $I R R=1.03,95 \% \mathrm{CI}=0.85-1.26$ ). Use of psychotropic medications was also associated with an increased risk of a fracture in females (relative risk [RR] 2.54; CI 1.57 - 4.11; p < 0.0001) but not in males (RR $=0.66 ; \mathrm{p}=0.584$; CI $0.15-2.86$ ). In both analyses, body mass index (BMI) was found to be the only confounding variable. After adjusting for BMI, the IRR in females decreased to 1.22 (95\% CI 1.02 1.45; $\mathrm{p}<0.015$ ) for falls and the RR decreased to 1.92 ( $\mathrm{p}<0.015$, CI 1.13 - 3.24) for fractures.

Gallagher et al. [16] have validated a comprehensive screening tool that enables the prescribing physician to assess an older patient's prescription drugs in the context of his/her concurrent diagnoses. Inter-rater reliability is favourable [17] with a kappa-coefficient of 0.75 for STOPP and 0.68 for START (Table 2).

STOPP (Screening Tool of Older Person's Prescriptions) encompasses 65 clinically significant criteria for potentially inappropriate prescribing in older people. Each criterion is linked with a concise explanation as to why the prescribing practice is potentially inappropriate. START (Screening Tool to Alert doctors to Right 
- The Screening Tool of Older Persons’ potentially inappropriate prescriptions (STOPP) classifies 65 common drug issues that contribute to inappropriate prescribing in the elderly.

- International studies using STOPP criteria indicate high potentially inappropriate medication prevalence rates.

- $\quad$ START (Screening Tool to Alert doctors to Right Treatment) consists of 22 evidence-based prescribing indicators for commonly encountered diseases in older people.

Treatment) consists of 22 evidence-based prescribing indicators for commonly encountered diseases in older people.

International studies using STOPP criteria worryingly indicate high potentially inappropriate medication (PIM) prevalence rates. A PIM index is calculated by dividing the total number of PIMs by the total number of medications. A UK study [29] carried out within an acute hospital setting found that the admission PIM prevalence was 26.7\% (95\% CI 20.5 - 32.9; 52 patients, 74 PIMs). The most common PIM categories on admission were central nervous system and psychotropic drugs, drugs adversely affecting patients at risk of falls and drugs acting on the urogenital system. The likelihood of having a PIM on admission was doubled in patients receiving more than ten medications compared with those taking fewer (odds ratio 2.3 [95\% CI $1.2-4.4$ ]; $\mathrm{p}=0.01$ ). The study reported a discharge PIM prevalence of $22.6 \%$ (95\% CI 16.7 - 28.5; 44 patients, 51 PIMs), with a significant reduction of PIMs on discharge $(\mathrm{p}=0.005)$.

Wahab et al. [30] assessed the prevalence and nature of pre-admission inappropriate prescribing by using the STOPP criteria amongst a sample of hospitalised elderly inpatients in South Australia. The total number of pre-admission medications screened during the study period was 949 and the median number of medicines per patient was nine (range 2 - 28). Overall the STOPP criteria identified 138 PIMs in 60\% patients. The most frequent PIM was for opiates prescribed in patients with recurrent falls (12.3\%). The other two culprit medicines were benzodiazepines in fallers (10.1\%) and proton pump inhibitors prescribed for peptic ulcer disease for long-term at maximum doses (9.4\%). The number of medications being taken had a positive correlation with pre-admission PIM use $(\mathrm{r}(\mathrm{s})=0.49, \mathrm{p}<0.01)$.

Medication review for patients may be undertaken by various health professionals. A randomised control trial [31] assessed the effect of clinical medication review undertaken by a pharmacist for elderly people living in care homes. This included 661 residents aged 65 years and over, on one or more medicines. The study found that general practitioners did not review most care home patients' medication. Medication changes initiated and proposed by the pharmacist were usually accepted, with a reduction in number of falls, but without any changes to overall drug costs. In addition, there were no significant changes in consultations, hospitalisations, mortality, standardised mini mental state examination or Barthel scores (Barthel Index consists of 10 items that measure a person's daily functioning, specifically the activities of daily living).

Wilcock et al. [32] utilised community pharmacists to collate data for their cross sectional survey which included a study population of 581 residents recruited from eighteen UK residential homes. They found that the use of psychotropic drugs was common, and observed a trend for increase in prescribing rates as compared to data from a previous survey in 2001. There was limited use of Calcium and Vitamin D supplements, which have the potential for reducing morbidity associated with falls. Although use of both calcium and vitamin D had increased significantly ( $8.3 \%$ in 2005 vs. $2.1 \%$ in 2001 ), overall this was relatively low with an eight percentage usage level in this at risk population (Table 3).

In their paper on prevention of falls and consequent injuries in elderly people, Kannus et al. [3] discuss the traditional approach often used for prevention of bone fracture injury with focus on prevention and treatment of osteoporosis. The aims are to maximise peak bone mass and prevent ongoing bone loss with regular exercise, calcium and vitamin D and treatment of osteoporosis with pharmacological medicines [15]. Service development in the UK NHS has included pharmacist-led, multidisciplinary team-supported, medication management clinics that focus on osteoporosis, falls and fracture prevention [33]. The pharmaceutical care approach [34] [35] utilised in these clinics within the secondary care setting, provide a structured framework to help identify polypharmacy issues and ensure safe and efficacious medication usage by patients. Assessment of compliance and adherence with medication taking [36] is undertaken by the pharmacist, with patients encouraged to raise their medication and health related concerns, and where the aims are to agree to an ongoing management plan [37] [38]. 
Table 3. Medication review.

- Medication review for patients may be undertaken by various health professionals.

- Clinical medication review by a pharmacist for older people in care home settings is associated with reduced falls risk.

- A traditional approach often used for falls and bone fracture injury prevention is management of osteoporosis risk.

Limitations for the findings reported from this review include study of publications identified via a search utilising only the PubMed database. Our work was undertaken as a quick, time-limited, preliminary fact finding exercise to understand what the current evidence base is for clinical medication review and falls in older people. Readers may find that the findings reported in this paper provides useful knowledge, and that our work helps inform wider database searches and helps inform more research activity in this area.

Tables 1-3 highlight the main knowledge generated from this review. These data will help inform our work as we move forward with the designing of a pharmacist delivered clinical medication review service for elderly fallers. This service would be part of the larger multi-disciplinary team supported integrated care pathway. In clinical practice elderly fallers often have numerous co-morbidities and are on multiple medicines. We aim to develop a research and evaluation strategy incorporating improvement science methodology [39], that generates more knowledge for medication related falls risk, with use of the STOPP/START tools as a starting point. It will be important also to remember that for delivery of effective pharmaceutical care, elderly people with age related deficits may benefit more from discontinuation and de-prescribing of some of their medications. This decision needs to be made considering all the medicines taken, and where the elderly patient reports side effects, and not only for medicines that are already defined as causing adverse events and therefore on the STOPP list.

\section{Conclusion}

This paper reports the current knowledge from the evidence base for clinical medication review and falls in the elderly. Both medication over-use and under-use occur frequently and may be harmful; many drugs commonly used by older persons have not been systematically studied as risk factors for falls. The STOPP START tools, validated for assessment of potentially inappropriate prescribing in the elderly, offer the possibility of provision of a structured clinical medication review to patients. There is a need for more research on the impact of these interventions on both the rates of falls and risk of falls in the elderly.

\section{Acknowledgements}

Research \& Development Department, Academic Directorate, London North West Healthcare NHS Trust for supporting this work.

\section{Financial Support}

This research received no specific grant from any funding agency, commercial or not-for-profit sectors.

\section{Conflict of Interest}

None.

\section{References}

[1] Department of Health (2009) The Prevention Package for Older People. http://webarchive.nationalarchives.gov.uk/20130107105354/http:/www.dh.gov.uk/en/Publicationsandstatistics/Publicat ions/dh_103146

[2] World Health Organisation (2012) Falls. WHO Factsheet No 344. http://www.who.int/mediacentre/factsheets/fs344/en/

[3] Kannus, P., Sievänen, H., Palvanen, M., Järvinen, T. and Parkkari, J. (2005) Prevention of Falls and Consequent Injuries in Elderly People. Lancet, 366, 1885-1893. http://dx.doi.org/10.1016/S0140-6736(05)67604-0

[4] Centers for Disease Control and Prevention (CDC) (2006) Fatalities and Injuries from Falls among Older AdultsUnited States, 1993-2003 and 2001-2005. Morbidity and Mortality Weekly Report, 55, 1221-1224.

[5] Rubenstein, L.Z. (2006) Falls in Older People: Epidemiology, Risk Factors and Strategies for Prevention. Age and 
Ageing, 35, 37-41. http://dx.doi.org/10.1093/ageing/afl084

[6] Cameron, I.D., Gillespie, L.D., Robertson, M.C., Murray, G.R., Hill, K.D., Cumming, R.G. and Kerse, N. (2012) Interventions for Preventing Falls in Older People in Care Facilities and Hospitals. Cochrane Database of Systematic Reviews, No. 12, Article No.: CD005465. http://dx.doi.org/10.1002/14651858.CD005465.pub3

[7] Lin, F.R. and Ferrucci, L. (2012) Hearing Loss and Falls among Older Adults in the United States. Archives of Internal Medicine, 172, 369-371. http://dx.doi.org/10.1001/archinternmed.2011.728

[8] Viljanen, A., Kaprio, J., Pyykkö, I., Sorri, M., Koskenvuo, M. and Rantanen, T. (2009) Hearing Acuity as a Predictor of Walking Difficulties in Older Women. Journal of the American Geriatrics Society, 57, 2282-2286. http://dx.doi.org/10.1111/j.1532-5415.2009.02553.x

[9] Skalska, A., Wizner, B., Piotrowicz, K., Klich-Rączka, A., Klimek, E., Mossakowska, M., et al. (2013) The Prevalence of Falls and Their Relation to Visual and Hearing Impairments among a Nation-Wide Cohort of Older Poles. Experimental Gerontology, 48, 140-146. http://dx.doi.org/10.1016/j.exger.2012.12.003

[10] Crowe, K. (2013) Increasing Isolation in a Confusing World: Dementia with Age Related Hearing Impairment. Old Age Psychiatrist, 55.

[11] Department of Health (2009) Hip Fracture including the Secondary Prevention of Further Fractures Related to Falls and Bone Fragility. http://www.pathwaysforhealth.org/application/render08.asp?reference=E866CB3073EF4E0CA4289A3A0C20407E

[12] Laybourne, A.H., Martin, F.C., Whiting, D.G. and Lowton, K. (2011) Could Fire and Rescue Services Identify Older People at Risk of Falls? Primary Health Care Research \& Development, 12, 395-399. http://dx.doi.org/10.1017/S1463423611000120

[13] British Geriatrics Society (2014) Fit for Frailty. Consensus Best Practice Guidance for the Care of Older People Living with Frailty in Community and Outpatient Settings. British Geriatrics Society in Association with the Royal College of General Practitioners and Age UK. http://www.bgs.org.uk/campaigns/fff/fff full.pdf

[14] Robb, E., Jarman, B., Suntharalingam, G., Higgens, C., Tennant, R. and Elcock, K. (2010) Using Care Bundles to Reduce In-Hospital Mortality: Quantitative Survey. BMJ, 340, c1234. http://dx.doi.org/10.1136/bmj.c1234

[15] NICE (2013) Falls in Older People: Assessing Risk and Prevention. Guidelines CG161. http://www.nice.org.uk/guidance/cg161/chapter/recommendations

[16] Gallagher, P., Ryan, C., Byrne, S., Kennedy, J., O’Mahony, D., STOPP (Screening Tool of Older Person’s Prescriptions) and START (Screening Tool to Alert Doctors to Right Treatment) (2008) Consensus Validation. International Journal of Clinical Pharmacology and Therapeutics, 46, 72-83. http://dx.doi.org/10.5414/CPP46072

[17] Gallagher, P., Baeyens, J.P., Topinkova, E., Madlova, P., Cherubini, A., Gasperini, B., et al. (2009) Inter-Rater Reliability of STOPP (Screening Tool of Older Persons' Prescriptions) and START (Screening Tool to Alert Doctors to Right Treatment) Criteria amongst Physicians in Six European Countries. Age and Ageing, 38, 603-606. http://dx.doi.org/10.1093/ageing/afp058

[18] Bjelakovic, G., Gluud, L.L., Nikolova, D., Whitfield, K., Wetterslev, J., Simonetti, R.G., et al. (2014) Vitamin D Supplementation for Prevention of Mortality in Adults. Cochrane Database of Systematic Reviews, No. 1, Article ID: CD007470.

[19] Gillespie, L.D., Robertson, M.C., Gillespie, W.J., Sherrington, C., Gates, S., Clemson, L.M. and Lamb, S.E. (2012) Interventions for Preventing Falls in Older People Living in the Community. Cochrane Database of Systematic Reviews, No. 9, Article ID: CD007146. http://dx.doi.org/10.1002/14651858.CD007146.pub3

[20] Beer, C., Hyde, Z., Almeida, O.P., Norman, P., Hankey, G.J., Yeap, B.B. and Flicker, L. (2011) Quality Use of Medicines and Health Outcomes among a Cohort of Community Dwelling Older Men: An Observational Study. British Journal of Clinical Pharmacology, 71, 592-599. http://dx.doi.org/10.1111/j.1365-2125.2010.03875.X

[21] Gnjidic, D., Hilmer, S.N., Blyth, F.M., Naganathan, V., Waite, L., Seibel, M.J., et al. (2012) Polypharmacy Cutoff and Outcomes: Five or More Medicines Were Used to Identify Community-Dwelling Older Men at Risk of Different Adverse Outcomes. Journal of Clinical Epidemiology, 65, 989-995. http://dx.doi.org/10.1016/j.jclinepi.2012.02.018

[22] Hartikainen, S., Lönnroos, E. and Louhivuori, K. (2007) Medication as a Risk Factor for Falls: Critical Systematic Review. Journals of Gerontology Series A: Biological Sciences and Medical Sciences, 62, 1172-1181. http://dx.doi.org/10.1093/gerona/62.10.1172

[23] Veal, F.C., Bereznicki, L.R., Thompson, A.J. and Peterson, G.M. (2014) Pharmacological Management of Pain in Australian Aged Care Facilities. Age and Ageing, 43, 851-856. http://dx.doi.org/10.1093/ageing/afu072

[24] Nishtala, P.S., Narayan, S.W., Wang, T. and Hilmer, S.N. (2014) Associations of Drug Burden Index with Falls, General Practitioner Visits, and Mortality in Older People. Pharmacoepidemiology and Drug Safety, 23, 753-758. http://dx.doi.org/10.1002/pds.3624 
[25] Berthoux, E., Dufour, C., Raharisondraibe, E. and Bonnefoy, M. (2013) Preventable Drug Events in Acute Geriatric Unit. Gériatrie et Psychologie Neuropsychiatrie du Vieillissement, 11, 15-20.

[26] Rhalimi, M., Helou, R. and Jaeker, P. (2009) Medication Use and Increased Risk of Falls in Hospitalized Elderly Patients: A Retrospective, Case-Control Study. Drugs \& Aging, 26, 847-852. http://dx.doi.org/10.2165/11317610-000000000-00000

[27] Payne, R.A., Abel, G.A., Simpson, C.R. and Maxwell, S.R. (2013) Association between Prescribing of Cardiovascular and Psychotropic Medications and Hospital Admission for Falls or Fractures. Drugs \& Aging, 30, 247-254. http://dx.doi.org/10.1007/s40266-013-0058-z

[28] Vitry, A.I., Hoile, A.P., Gilbert, A.L., Esterman, A. and Luszcz, M.A. (2010) The Risk of Falls and Fractures Associated with Persistent Use of Psychotropic Medications in Elderly People. Archives of Gerontology and Geriatrics, 50, e1-e4. http://dx.doi.org/10.1016/j.archger.2009.04.004

[29] Onatade, R., Auyeung, V., Scutt, G. and Fernando, J. (2013) Potentially Inappropriate Prescribing in Patients on Admission and Discharge from an Older Peoples' Unit of an Acute UK Hospital. Drugs \& Aging, 30, 729-737. http://dx.doi.org/10.1007/s40266-013-0097-5

[30] Wahab, M.S., Nyfort-Hansen, K. and Kowalski, S.R. (2012) Inappropriate Prescribing in Hospitalised Australian Elderly as Determined by the STOPP Criteria. International Journal of Clinical Pharmacy, 34, 855-862. http://dx.doi.org/10.1007/s11096-012-9681-8

[31] Zermansky, A.G., Alldred, D.P., Petty, D.R., Raynor, D.K., Freemantle, N., Eastaugh, J. and Bowie, P. (2006) Clinical Medication Review by a Pharmacist of Elderly People Living in Care Homes-Randomised Controlled Trial. Age and Ageing, 35, 586-591. http://dx.doi.org/10.1093/ageing/afl075

[32] Wilcock, M., MacMahon, D. and Woolf, A. (2005) Use of Medicines That Influence Falls or Fractures in a Residential Home Setting. Pharmacy World and Science, 27, 220-222. http://dx.doi.org/10.1007/s11096-004-3707-9

[33] Tanna, N. (2004) Care of the Elderly-An Osteoporosis Medication Management Clinic. Hospital Pharmacist, 11, 231-238.

[34] Cipolle, R.J., Strand, L.M. and Morley, P.C. (1998) Pharmaceutical Care Practice. McGraw-Hill, Health Professions Division, New York.

[35] Cipolle, R.J., Strand, L.M. and Morley, P.C. (2012) Pharmaceutical Care Practice: The Patient Centered Approach to Medication Management. 3rd Edition, McGraw-Hill, Health Professions Division, New York.

[36] NICE (2009) Guidelines CG76. Medicines Adherence. http://pathways.nice.org.uk/pathways/medicines-adherence/medicines-adherence-overview\#content=view-node\%3Ano des-reviewing-medicines

[37] Tanna, N., Batten, C., Pitkin, J., Rogers, P. and Kelham, C. (2005) Shared Decision Making and Care Planning with Patients. The Journal of the British Menopause Society, 11, 180.

[38] Tanna, N., Batten, C., Pitkin, J., Rogers, P. and Kelham, C. (2007) Evaluation of a Medication Management Clinic Service, Using Action Research Methodology. Osteoporosis International, 18, S310.

[39] Marshall, M., Pronovost, P. and Dixon-Woods, M. (2013) Promotion of Improvement as a Science. The Lancet, 381, 419-421. http://dx.doi.org/10.1016/S0140-6736(12)61850-9 